EPJ Web of Conferences 49, 15006 (2013)

DOI: $10.1051 /$ epjconf/20134915006

(C) Owned by the authors, published by EDP Sciences, 2013

\title{
Searches for new physics with tau leptons at the ATLAS detector
}

\author{
K.J.C. Leney ${ }^{1, a}$ on behalf of the ATLAS Collaboration \\ ${ }^{1}$ University of the Witwatersrand, Johannesburg, South Africa
}

\begin{abstract}
Searches for heavy particles decaying to a pair of tau leptons, and searches for pair produced thirdgeneration scalar leptoquarks decaying to a b-jet and a tau lepton are reported. The studies use the full data sample recorded in 2011 at $\sqrt{s}=7 \mathrm{TeV}$ centre-of-mass energy by the ATLAS experiment at the LHC.
\end{abstract}

\section{Introduction}

New heavy gauge bosons are predicted by many beyond the Standard Model theories [1-6]. Several models with extended weak or hypercharge gauge groups that offer an explanation for the high mass of the top quark predict that such bosons couple preferentially to third-generation fermions [7]. Decays to pairs of tau leptons are therefore of particular importance. The Sequential Standard Model (SSM) is used as the benchmark model. It contains a heavy neutral gauge boson, $Z_{\mathrm{SSM}}^{\prime}$, with the same couplings to fermions as the SM Z-boson. Tau leptons can decay either leptonically (to an electron or muon plus two neutrinos), or hadronically (typically to one or three charged hadrons, plus one neutrino, and zero to four neutral hadrons) - denoted $\tau_{\text {had. }}$. Since the final state includes two taus, this leads to three possible sub-categories of events: di-lepton (branching fraction $=12.42 \%$ ), lepton-hadron (branching fraction $=45.64 \%$ ) and hadron-hadron (branching fraction $=41.94 \%)$. The $e \mu, e \tau_{\text {had }}, \mu \tau_{\text {had }}$, and $\tau_{\text {had }} \tau_{\text {had }}$ decay modes are considered [8].

Leptoquarks (LQ) are colour-triplet bosons that carry both lepton and baryon numbers and have a fractional electric charge. They are predicted by many extensions of the Standard Model (SM) [9-15] and may provide unification between the quark and lepton sectors. In accordance with experimental results on lepton-number violation, flavour-changing neutral currents and proton decay, it is assumed that individual leptoquarks do not couple to particles from different generations [16, 17], thus leading to three generations of leptoquarks. ATLAS searches for pair-produced, scalar, third generation leptoquarks $\left(\mathrm{LQ}_{3}\right)$ are summarised. Both leptoquarks are assumed to decay into a tau lepton and a $b$-quark, leading to a $\tau b \tau b$ final state. The branching fraction of $\mathrm{LQ}_{3}$ decays to $\tau b$ is assumed to be equal to $100 \%$. Only the lepton-hadron decay mode is considered, resulting in either an $e \tau_{\text {had-vis }} b b+3 v$ or $\mu \tau_{\text {had-vis }} b b+3 v$ final state, where $\tau_{\text {had-vis }}$ is the visible (non-neutrino) components of the hadronic tau decay.

\footnotetext{
a e-mail: katharine.leney @ cern.ch
}

All results presented here are based on a total integrated luminosity of $4.7 \mathrm{fb}^{-1}$ of proton-proton collision data at a centre-of-mass energy of $\sqrt{s}=7 \mathrm{TeV}$, collected by the ATLAS detector at the LHC during 2011.

\section{The ATLAS detector}

The ATLAS detector [18] is a multi-purpose detector with a forward-backward symmetric cylindrical geometry and nearly $4 \pi$ coverage in solid angle. ATLAS uses a righthanded coordinate system with its origin at the nominal interaction point (IP) and the $z$-axis along the beam pipe. The $x$-axis points from the IP to the centre of the LHC ring, and the $y$-axis points upward. Cylindrical coordinates $(r, \phi)$ are used in the transverse $(x, y)$ plane, with $\phi$ being the azimuthal angle around the beam pipe. The pseudorapidity $\eta$ is defined in terms of the polar angle $\theta$ by $\eta=-\ln (\tan (\theta / 2))$. The three major sub-components of ATLAS are the tracking detector, the calorimeter and the muon spectrometer.

\section{$3 \mathbf{Z}^{\prime} \rightarrow \tau \tau$ analysis}

\subsection{Event selection}

Events in the $e \mu$ channel are required to pass a single muon trigger with a transverse momentum $\left(p_{\mathrm{T}}\right)$ threshold of $18 \mathrm{GeV}$. Selected events must contain exactly one isolated muon with $p_{\mathrm{T}}>25 \mathrm{GeV}$ and one isolated electron with $p_{\mathrm{T}}>35 \mathrm{GeV}$. The electron and muon must have opposite-sign charge, and no additional electrons (muons) with $p_{\mathrm{T}}>15(10) \mathrm{GeV}$ are allowed. In order to reject contributions from top quark backgrounds, events may not have more than one jet $\left(p_{\mathrm{T}}>25 \mathrm{GeV}\right)$. The missing transverse energy typically points away from the highest$p_{\mathrm{T}}$ lepton in signal events, so the angle in the transverse plane between the highest- $p_{\mathrm{T}}$ lepton and the missing transverse energy $\left(E_{\mathrm{T}}^{\mathrm{miss}}\right)$ is required to be $<2.6$ radians. The two leptons are required to be back-to-back by demanding that:

$$
p_{\hat{\zeta}}^{v i s}\left(=\vec{p}_{\mathrm{T}}(e) \cdot \hat{\zeta}+\vec{p}_{\mathrm{T}}(\mu) \cdot \hat{\zeta}\right)<10 \mathrm{GeV}
$$


where $\hat{\zeta}$ is a unit vector along the bisector of the electron and muon momenta.

Events in the $e \tau_{\text {had }}$ and $\mu \tau_{\text {had }}$ channels are selected by requiring that they pass a single electron trigger with a $p_{\mathrm{T}}$ threshold in the range $20-22 \mathrm{GeV}$ or a single muon trigger with $p_{\mathrm{T}}$ threshold $18 \mathrm{GeV}$ respectively. Selected events must contain exactly one isolated electron with $p_{\mathrm{T}}>30 \mathrm{GeV}$ or exactly one isolated muon with $p_{\mathrm{T}}>25 \mathrm{GeV}$, and one hadronic 1-prong tau with $p_{\mathrm{T}}>35 \mathrm{GeV}$. Three-prong taus are not included because of the large fake rate in the $W+$ jets background. The lepton and tau must have opposite-sign charge and the angle in the transverse plane between them must be $<2.7$ radians. No additional electrons (muons) with $p_{\mathrm{T}}>15$ (4) $\mathrm{GeV}$ are allowed. In the $e \tau_{\text {had }}$ channel, $E_{\mathrm{T}}^{\text {miss }}$ is required to be $>30 \mathrm{GeV}$ (to reduce contributions from $Z / \gamma \rightarrow e e$ and multi-jet events) and the transverse mass between the electron and the $E_{\mathrm{T}}^{\text {miss }}$ must be $<50 \mathrm{GeV}$ (to supress the $W+$ jets background).

Events in the $\tau_{\text {had }} \tau_{\text {had }}$ channel are required to pass either a di-tau trigger with a transverse energy $\left(E_{\mathrm{T}}\right)$ threshold of (20) $29 \mathrm{GeV}$ for the (sub-)leading tau or a single tau trigger with $E_{\mathrm{T}}>125 \mathrm{GeV}$. Selected events must have exactly two hadronic tau candidates with $p_{\mathrm{T}}>50 \mathrm{GeV}, 1$ - or 3-prongs, and opposite-sign charge to each other. No additional electrons (muons) with $p_{\mathrm{T}}>15(10) \mathrm{GeV}$ are allowed. The two taus are required to be back-to-back, with the angular separation in the transverse plane larger than 2.7 radians.

\subsection{Background estimation}

The dominant background processes in the $e \mu$ channel are $t \bar{t}, Z / \gamma \rightarrow \tau \tau$, and di-boson production. Contributions from other $W / Z+$ jets processes are very small in the signal region. All electroweak and top backgrounds are estimated using Monte Carlo (MC) simulations and checked in high purity control regions. Multi-jet events are suppressed by making stringent requirements on the lepton isolation and their contribution is shown to be negligible using data-driven methods.

The main background processes in the $e \tau_{\text {had }}$ and $\mu \tau_{\text {had }}$ channels are $Z / \gamma \rightarrow \tau \tau, W+$ jets, $t \bar{t}$, and di-boson. Single top-quark, $Z / \gamma \rightarrow e e / \mu \mu$, and multi-jet processes contribute to a lesser degree. The contributions from $W+$ jets and multi-jet processes are modelled by deriving fake-factors to parameterise the rate at which jets may pass the hadronic tau identification, and for lepton $(e \mu)$ candidates in jets to pass the lepton isolation requirements. Other background processes are estimated from MC.

The dominant background processes to the $\tau_{\text {had }} \tau_{\text {had }}$ channel are $Z / \gamma \rightarrow \tau \tau$ and multi-jet production. The $W \rightarrow \tau v+$ jets provides a minor contribution, and the small contributions from other background processes $(Z / \gamma \rightarrow e e / \mu \mu, W \rightarrow e / \mu v+\mathrm{jets}, t \bar{t}$, single top-quark, and di-boson) are also considered. The shape of the multi-jet contribution is extracted from a fit to events where the hadronic-tau candidates have the same-sign charge. This is normalised to the low total transverse mass $\left(m_{\mathrm{T}}^{\text {tot }}\right)$ sideband region, where:

$$
m_{T}^{t o t}=\sqrt{2\left(p_{\mathrm{T}}^{\tau_{1}} p_{\mathrm{T}}^{\tau_{2}} C+E_{\mathrm{T}}^{\mathrm{miss}} p_{\mathrm{T}}^{\tau_{1}} C_{1}+E_{\mathrm{T}}^{\mathrm{miss}} p_{\mathrm{T}}^{\tau_{2}} C_{2}\right)},
$$

where $\quad C=1-\cos \Delta \phi\left(\tau_{1}, \tau_{2}\right), \quad C_{1(2)}=1-$ $\cos \Delta \phi\left(\tau_{1(2)}, E_{\mathrm{T}}^{\mathrm{miss}}\right)$, and $\tau_{1(2)}$ is the first (second) tau visible decay products. Other background processes are estimated from MC.

\subsection{Results}

A $Z^{\prime}$-boson would typically have a narrow width $(\sim 3 \%$ of the $Z^{\prime}$ mass $\left(M_{Z^{\prime}}\right)$, but the large $E_{\mathrm{T}}^{\text {miss }}$ from neutrinos in tau decays causes significant degradation. Events in the signal region are therefore required to have a large $m_{\mathrm{T}}^{\text {tot }}$ and a simple counting experiment is performed across all analysis channels. The final $m_{\mathrm{T}}^{\text {tot }}$ requirement is optimised in each channel for each mass hypothesis. The final $m_{\mathrm{T}}^{\text {tot }}$ distributions are shown in Fig. 1.

Event yields after applying a cut on the $m_{\mathrm{T}}^{\text {tot }}$ are given in Table 1. All sources of systematic uncertainty are considered. The dominant systematic uncertainty on the signal is the hadronic tau identification efficiency (typically $10-15 \%)$. The main sources of systematic uncertainty on the backgrounds are the tau energy scale, and uncertainties related to the data-driven background estimation methods.

The number of observed events is consistent with the Standard Model expectation across all channels, and upper limits are therefore set on the production of a highmass resonance decaying to tau-pairs. When all channels are combined, the SSM $Z^{\prime} \rightarrow \tau \tau$ process is excluded at 95\% confidence level (CL) for $Z^{\prime}$ masses up to $1.4 \mathrm{TeV}$ (the expected limit is $1.42 \mathrm{TeV}$ ). Limits for the individual channels and the combination are shown in Fig. 2.

\section{$4 \mathbf{L Q}_{3} \mathbf{L Q}_{3} \rightarrow \tau b \tau b$ analysis}

\subsection{Event selection}

Events are required to have exactly one reconstructed electron (or muon) with $p_{\mathrm{T}}>25(20) \mathrm{GeV}$, and exactly one hadronic tau decay candidate with $p_{\mathrm{T}}>30 \mathrm{GeV}$ with opposite-sign charge to the lepton. The $E_{\mathrm{T}}^{\mathrm{miss}}$ is required to be larger than $20 \mathrm{GeV}$ in order to reject multi-jet and $Z / \gamma \rightarrow e e / \mu \mu$ processes. At least two jets are required, with the leading jet having $p_{\mathrm{T}}>50 \mathrm{GeV}$ and the subleading jet having $p_{\mathrm{T}}>25 \mathrm{GeV}$. The signal-to-background ratio is improved by requiring that either the leading or sub-leading jet passes the $b$-tagging requirements.

The visible mass $\left(m_{\tau_{\text {had-vis }}-\text { jet }}\right)$ of the tau candidate and the closest jet in $\eta-\phi$ space (minimum $\Delta R$ ) is required to be larger than $90 \mathrm{GeV}$. Only jets with $p_{\mathrm{T}}>40 \mathrm{GeV}$ are considered. This cut rejects semi-leptonic $t \bar{t}$ events where the tau candidate is faked by jets from $W \rightarrow q \bar{q}$ decays.

The $E_{\mathrm{T}}^{\text {miss }}$ in leptoquark events arises from neutrinos accompanying the tau decays. Taus originating from leptoquarks typically have high momentum, thus the decay 


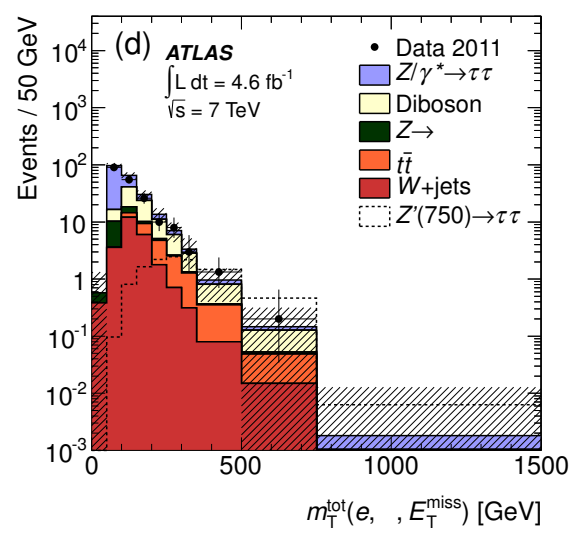

(a) $e \mu$ Channel

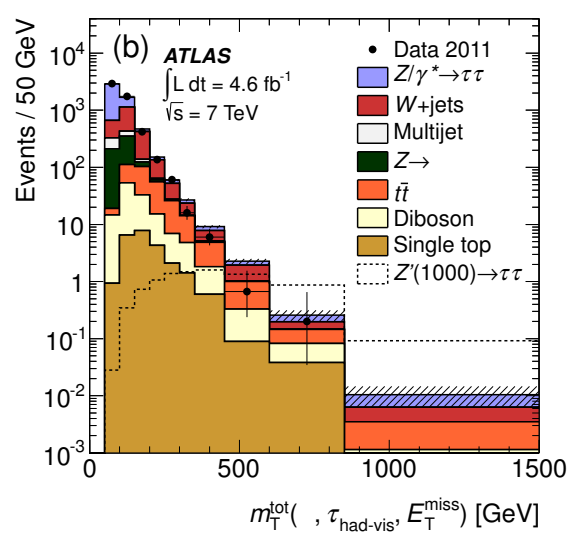

(c) $\mu \tau_{\text {had }}$ Channel

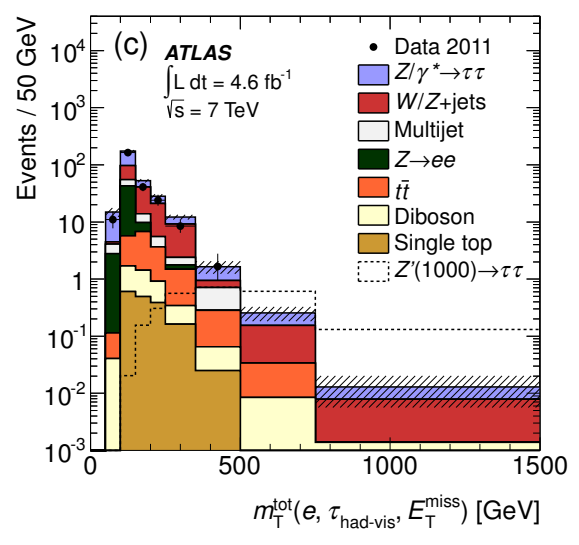

(b) $e \tau_{\text {had }}$ Channel

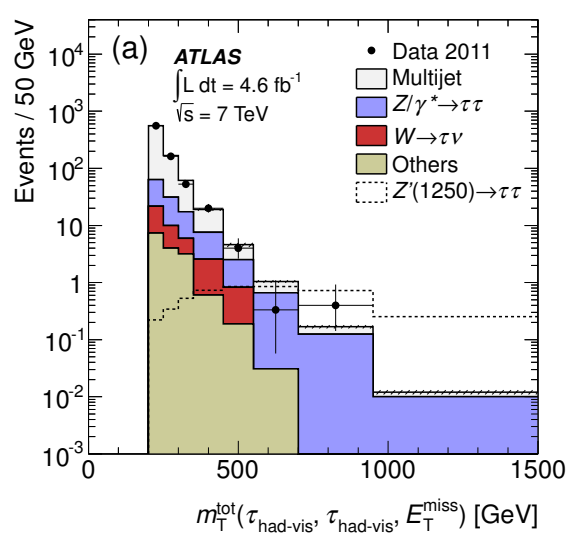

(d) $\tau_{\text {had }} \tau_{\text {had }}$ Channel

Figure 1. Data and MC comparisons of the $m_{\mathrm{T}}^{\text {tot }}$ variable after applying all cuts in the (a) $e \mu$, (b) $e \tau_{\text {had }}$, (c) $\mu \tau_{\text {had }}$, and (d) $\tau_{\text {had }} \tau_{\text {had }}$ channels [8].

Table 1. Expected and observed number of events in each channel after applying a cut on the $m_{\mathrm{T}}^{\text {tot }}$ (yields given for mass hypothesis closest to exclusion limit) [8].

\begin{tabular}{lcccc}
\hline & $e \mu$ & $e \tau_{\text {had }}$ & $\mu \tau_{\text {had }}$ & $\tau_{\text {had }} \tau_{\text {had }}$ \\
\hline Exp Background & $3.6 \pm 0.4$ & $1.6 \pm 0.5$ & $1.4 \pm 0.4$ & $1.0 \pm 0.3$ \\
Exp Signal & $6.7 \pm 0.3$ & $5.0 \pm 0.5$ & $5.5 \pm 0.7$ & $6.3 \pm 1.1$ \\
Observed & 5 & 0 & 1 & 2 \\
\hline
\end{tabular}

products are predominantly collinear and the $E_{\mathrm{T}}^{\text {miss }}$ direction is correlated with the direction of the visible tau decay products. Two variables are defined in order to improve the separation of signal and background: the absolute difference in $\phi$ between the charged lepton and $E_{\mathrm{T}}^{\text {miss }}\left(\left|\Delta \phi\left(E_{\mathrm{T}}^{\text {miss }}, \ell\right)\right|\right)$, and the absolute difference in $\phi$ between the tau candidate and $E_{\mathrm{T}}^{\text {miss }}\left(\left|\Delta \phi\left(E_{\mathrm{T}}^{\text {miss }}, \tau_{\text {had-vis }}\right)\right|\right)$. Events must satisfy the following requirement:

$$
\left.\left|\Delta \phi\left(E_{\mathrm{T}}^{\mathrm{miss}}, \ell\right)\right| \leq-\frac{1.5}{\pi}\left|\Delta \phi\left(E_{\mathrm{T}}^{\text {miss }}, \tau_{\text {had-vis }}\right)\right|+2 \text { (radians }\right)
$$

where $\ell=e, \mu$.

\subsection{Background estimation}

The dominant background process to the analysis is $t \bar{t}$ production. The $Z / \gamma \rightarrow \tau \tau$ and multi-jet processes also provide important contributions, with $W+$ jets, $Z / \gamma \rightarrow e e / \mu \mu$, di-boson, and single top-quark production providing minor contributions.

The multi-jet background contribution to the muon channel analysis is estimated using an ABCD method with the lepton-tau charge product and the lepton isolation as separating variables. Normalisation factors for electroweak and top backgrounds are calculated by defining background enriched control regions in data and performing a fit. The shapes of kinematic distributions are taken from data.

In the electron channel, the multi-jet shape and normalisation are determined from data by defining enriched control regions and fitting to get the scale. The shape of electroweak and top backgrounds is taken from MC and normalised based on the number of events in simulation and data. 


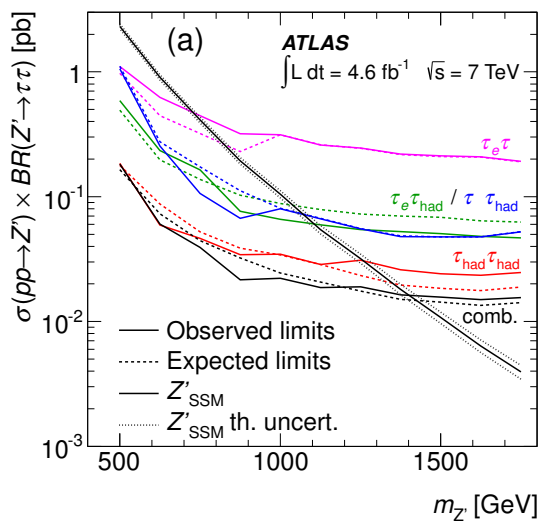

(a)

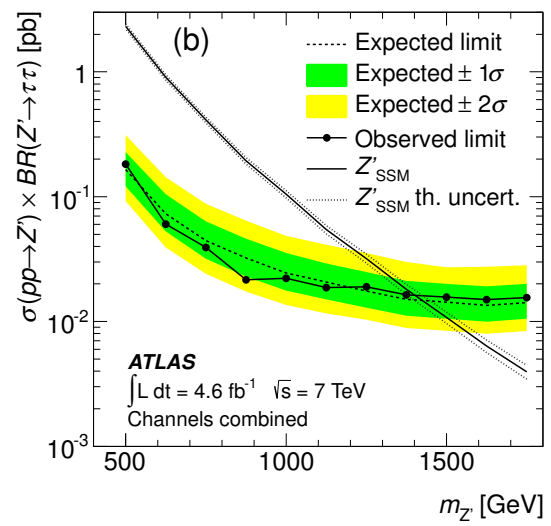

(b)

Figure 2. (a) The expected (dashed) and observed (solid) $95 \%$ credibility upper limits on the cross section times $\tau^{+} \tau^{-}$branching fraction, in the $e \mu, e \tau_{\text {had }}, \mu \tau_{\text {had }}$, and $\tau_{\text {had }} \tau_{\text {had }}$ channels and for the combination. The expected SSM $Z^{\prime}$ production cross section and its corresponding theoretical uncertainty (dotted) are also included. (b) The expected and observed limits for the combination including $1 \sigma$ and $2 \sigma$ uncertainty bands. $Z^{\prime}$ masses up to $1.40 \mathrm{TeV}$ are excluded, in agreement with the expected limit of $1.42 \mathrm{TeV}$ in the absence of a signal [8].

\subsection{Results}

The $S_{\mathrm{T}}$ variable is used to test for the existence of leptoquarks, where $S_{\mathrm{T}}$ is defined as the scalar sum of the $p_{\mathrm{T}}$ of the charged light lepton, the tau, the two highest- $p_{\mathrm{T}}$ jets and the $E_{\mathrm{T}}^{\text {miss }}$ in the event. The $S_{\mathrm{T}}$ distributions for both channels are shown in Fig. 3.

At very high $S_{\mathrm{T}}$, there is poor statistical precision for MC background processes. The sum of the background processes is fitted in the region $350 \mathrm{GeV}<S_{\mathrm{T}}<2000 \mathrm{GeV}$ to an exponential function using a maximum likelihood fit. In this way the distribution is smoothed and a background expectation is provided throughout this $S_{\mathrm{T}}$ region. Comparisons of the fitted distributions to data are shown in Fig. 4.

All sources of systematic uncertainty are considered. The dominant source of uncertainty on the backgrounds comes from methods used to determine the normalisation factors $(10-20 \%)$, while the main sources of uncertainty on the signal come from the identification of $b$-jets (15\%) and theory uncertainties on the production process (17\%).

Two alternative models are built to describe background-only and signal+background hypotheses. For each leptoquark mass hypothesis, a single 'signal strength' parameter $(\mu)$ multiplies the expected signal in each bin, where $\mu=0$ corresponds to the absence of a signal and $\mu=1$ corresponds to the presence of a signal with nominal strength. The model describes the expected number of signal $\left(s_{i}\right)$ and background $\left(b_{i}\right)$ events in each bin using a Poisson distribution. The statistical analysis of the data employs a binned likelihood function $\mathscr{L}(\mu, \theta)$. The likelihood in each channel is a product over bins in the $S_{\mathrm{T}}$ distributions defined as:

$$
\mathscr{L}(\mu, \theta)=\prod_{i=\text { bin }} \operatorname{Poisson}\left(N_{i} \mid \mu s_{i}+b_{i}\right),
$$

where $s_{i}$ and $b_{i}$ are the expected number of signal and background events in bin $i$ respectively, and $N_{i}$ is the observed number of events. Both $s_{i}$ and $b_{i}$ depend on nuisance parameters $\theta$. Pseudo-experiments are generated according to background-only and signal+background models to obtain distributions of the test statistic, $\log (\mathscr{L}(\mu, \theta) / \mathscr{L}(0, \theta))$. The $\mathrm{CL}_{\mathrm{s}}$ method [19] is used to calculate the $p$-values. The signal strength parameter is varied iteratively to find the $95 \%$ confidence level.

The resulting cross-section times branching fraction limits as a function of leptoquark mass are calculated. It is assumed that $B R\left(\mathrm{LQ}_{3} \rightarrow \tau b\right)=1.0$. The data are found to be consistent with the background-only hypothesis and third generation scalar leptoquarks are observed (expected) to be excluded at $95 \%$ confidence level for masses below $501(528) \mathrm{GeV}$ and $478(521) \mathrm{GeV}$ in the electron and muon channels respectively. When the channels are combined, third generation scalar leptoquark production is excluded at $95 \%$ confidence level for leptoquark masses up to $538 \mathrm{GeV}$ (the expected limit is $577 \mathrm{GeV}$ ). The 95\% CL upper bounds on the NLO cross-section for scalar leptoquark pair production as a function of mass when both channels are combined is shown in Fig. 5.

\section{Summary}

Searches for high mass resonances decaying to a pair of tau leptons, and for third generation scalar leptoquarks decaying to a tau lepton and a $b$-quark have been performed at ATLAS. No excess over the predicted SM background is observed in the data in either channel. The SSM $Z^{\prime} \rightarrow \tau \tau$ process is excluded for $Z^{\prime}$ masses up to $1.4 \mathrm{TeV}$. The assumption is made that $B R\left(\mathrm{LQ}_{3} \rightarrow \tau b\right)=100 \%$ and pair produced third generation scalar leptoquarks are excluded for leptoquark masses up to $525 \mathrm{GeV}$. Both results provide the most stringent limits to date on their respective models. 


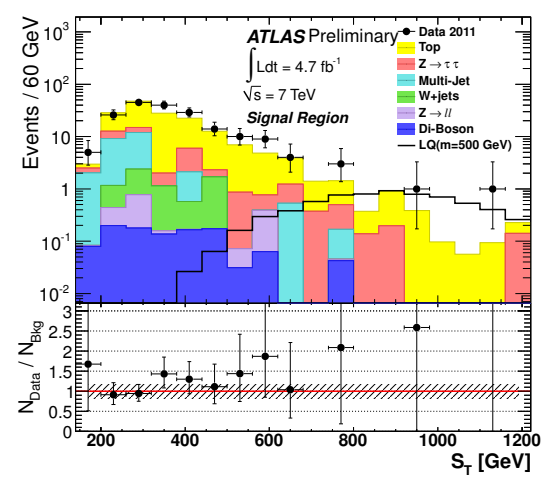

(a) Electron channel

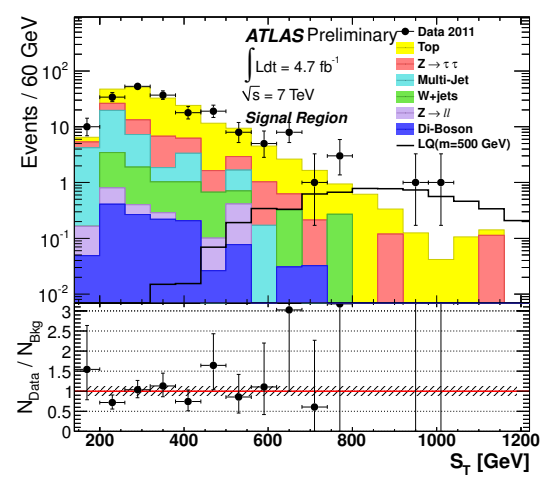

(b) Muon channel

Figure 3. Data and MC comparisons of the $S_{\mathrm{T}}$ variable after applying all cuts in the (a) electron and (b) muon channels [20].

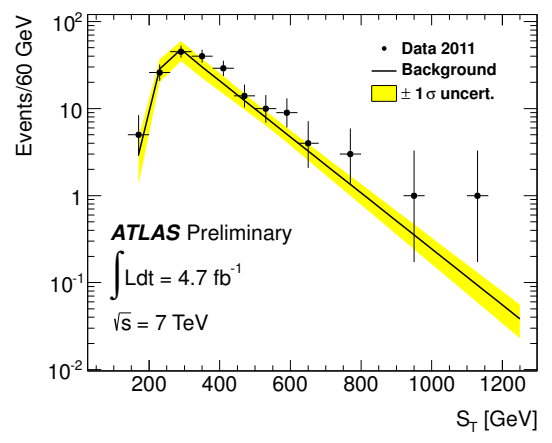

(a) Electron channel

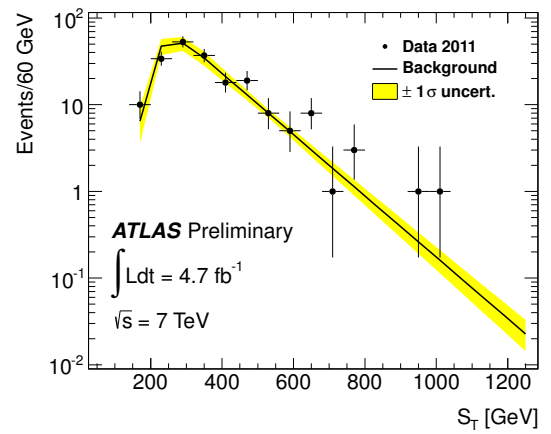

(b) Muon channel

Figure 4. Comparison of fitted $S_{\mathrm{T}}$ background shape to data in the (a) electron and (b) muon channels [20].

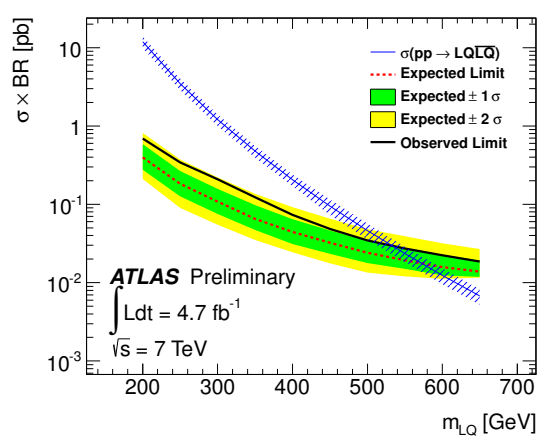

Figure 5. The combined 95\% CL upper limit on the pair production cross-section of third generation scalar leptoquarks as a function of leptoquark mass [20].

\section{References}

[1] P. Langacker, Rev. Mod. Phys. 81, 1199-1228 (2009)

[2] J. L. Hewitt et al, Phys. Rept. 183, 193-381 (1989)

[3] M. Cvetic et al, arXiv:hep-ph/9504216 (1995)

[4] A. Leike, Phys. Rept. 317, 143-250 (1999)

[5] G. G. Rizzo, arXiv:hep-ph/0610104 (2006)

[6] R. Diener et al, Phys. Rev. D 83, 115008 (2011)
[7] K. R. Lynch et al, Phys. Rev. D 63, 035006 (2001)

[8] ATLAS Collaboration, Submitted to Phys. Lett. B, arXiv:hep-ex/1210.6604 (2012)

[9] S. K. Dimopoulos and L. Susskind, Nucl. Phys. B 155, 237-252 (1979)

[10] S. K. Dimopoulos, Nucl. Phys. B 168, 62-92 (1980)

[11] E. J. Eichten and K. Lane, Phys. Lett. B 90, 125-130 (1980)

[12] V. D. Angelopoulos et al, Nucl. Phys. B 292, 50 (1986)

[13] W. Buchmüller and D. Wyler, Phys. Lett. B 177, 377$382(1986)$

[14] J. C. Pati and A. Salam, Phys. Rev. D 10, 275-289 (1974)

[15] H. Georgi and S. L. Glashow, Phys. Rev. Lett. 32, 438-441 (1974)

[16] W. Buchmüller, R. Rückl, and D. Wyler, Phys. Lett. B 191, 21 (1986)

[17] S. Kovalenko, and I. Schmidt, Phys. Lett. B 562, 104108 (2003)

[18] ATLAS Collaboration, JINST 3, S08003 (2008)

[19] A. L. Read, J. Phys. G28, 2693-2704 (2002)

[20] ATLAS Collaboration, CERN-PH-EP-2012-317, to be submitted to JHEP 